Platelet aggregation results, expressed as VN ARU were divided into tertiles:

- $\mathrm{T} 1$ (ARU 363 - 405) (n=76),

- T2 (ARU 406 - 436) ( $=76)$,

- T3 (ARU 437 - 596) ( $\mathrm{n}=72)$.

Figure 1, demonstrates that all-cause mortality is significantly different between groups, (log-rank, $\mathrm{P}=0.009)$, with higher ARU values being associated with increased mortality.

A co-regression analysis indicates that, ex-smoker status $(\mathrm{HR}=3.60,95 \% \mathrm{CI}[1.47-8.85] \mathrm{p}=0.005)$, in addition to $\mathrm{HPR}$ on presentation $(\mathrm{HR}=3.13,95 \% \mathrm{CI}[1.38-7.10] \mathrm{p}$ $=0.006)$, were statistically significant predictors of mortality at 10 years.

Conclusion Our study demonstrates that ARU values less than the previously defined cut off 550 are associated with reduced survival at 10 years.

There are currently a number of pharmacological strategies aimed at reducing the considerable ongoing ischaemic risk in patients admitted with NSTEMI, including prolonged dual antiplatelet therapy, prolonged monotherapy with a P2Y12 inhibitor or low dose rivaroxaban. In order to improve longterm prognosis, NSTEMI patients with suboptimal, but not strict HPR to aspirin, might benefit from alternative and/or adjunctive antithrombotic treatment options. We suggest that this hypothesis warrants further study.

Conflict of Interest None

\section{COMPARING THE LENGTH OF STAY, EFFICACY AND SAFETY OF A NEW 1 HOUR "RULE-OUT" PATHWAY TO STANDARD CARE FOR PATIENTS PRESENTING TO THE EMERGENCY DEPARTMENTS WITH A SUSPECTED ACUTE CORONARY SYNDROME AT BRIGHTON AND SUSSEX UNIVERSITY HOSPITALS NHS TRUST}

${ }^{1}$ Nicolas Buttinger*, ${ }^{2}$ Daniel White, ${ }^{1} J o n a t h a n$ Shurlock, 'Lucy Blows, ${ }^{1}$ Maureen Dooley. ${ }^{1}$ Brighton and Sussex University Hospitals NHS Trust; ${ }^{2}$ Royal Surrey County Hospital Guildford

10.1136/heartjnl-2019-BCS.147

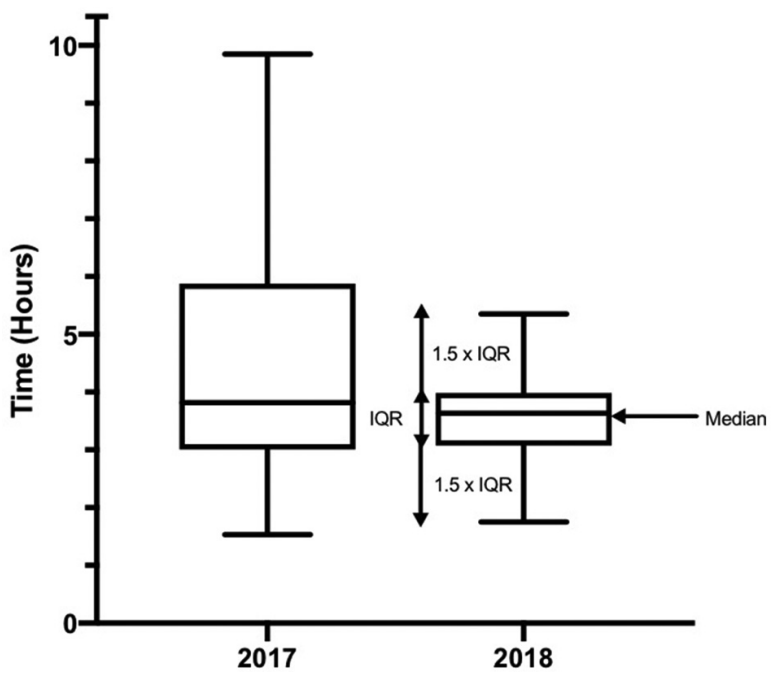

Abstract 150 Figure 1 Time from arrival to discharge home from the ED in all patients presenting with suspected ACS $(p=0.012)$

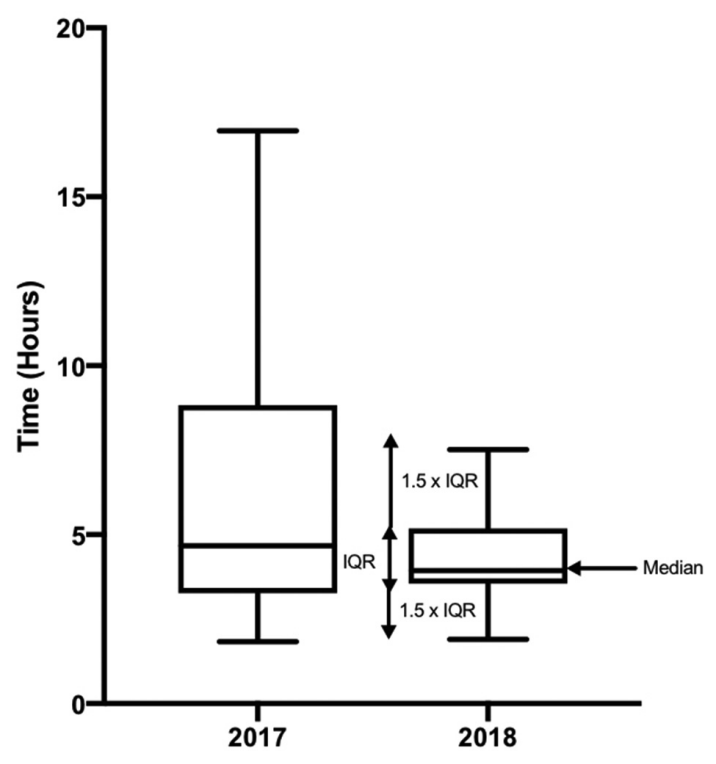

Abstract 150 Figure 2 Time from arrival to discharge home from the ED for patients with a To $>12 \mathrm{ng} / \mathrm{L}(\mathrm{p}=0.0359)$

Aim To compare the length of stay, efficacy and safety of a new $0 \mathrm{~h} / 1 \mathrm{~h}$ algorithm, utilising a high sensitivity troponin $\mathrm{T}$ (HsTnT) assay, with the previous standard 3 hour troponin at the Brighton and Sussex University Hospitals NHS Trust (BSUH) Emergency Departments (ED).

Methods The 2015 ESC guidelines approved a 1 hour algorithm used in conjunction with a HsTnT assay and clinical assessment to safely "rule-out" an NSTEMI in patients presenting to the ED with suspected ACS. We collected data on all patients attending the ED with suspected ACS in April 2017-pre and April 2018-post pathway implementation. The pathway triages patients into "rule-in", "rule-out" and "observation" groups. The initial pathway we developed included a lower cut-off than the ESC algorithm, $<3$ versus (v) $<5 \mathrm{ng} / \mathrm{L}$ for the initial troponin value (To) in order to allocate a patient to the "rule-out" group. 30-day all-cause mortality and major adverse cardiovascular events (MACE) were collected for both years. The final diagnosis was collected from discharge summaries for those admitted from the ED.

Results In April 2017 v April 2018 there were 391 v 491 patients who presented to the ED with suspected ACS. Mean age of 61 v 57 years and 59.3\% v 58.5\% male.

There was a reduction with the new pathway in the median time from ED arrival to discharge for all discharged patients 3.92 hours [IQR $=3.18-6.23]$ v 3.78 hours [IQR = $3.2-5.17]$. In patients triaged to the "rule-out" group, $281 \mathrm{v}$ 392 , the median time from ED arrival to discharge was 3.82 hours [IQR $=3-5.87]$ v 3.63 hours [IQR $=3.07-3.98$ ] (see figure 1). In those who had a To value $>12 \mathrm{ng} / \mathrm{L}, 63 \mathrm{v} 71$ patients, and subsequently discharged home, there was a significant reduction in the median time from ED arrival to discharge from 4.67 hours $[\mathrm{IQR}=3.27-8.83]$ to 3.93 hours [IQR = 3.55-5.18] (see figure 2).

In April 2018 65.6\%, 24.4\% and 10\% of patients with a suspected ACS were triaged to the "rule-out", "observation" and "rule-in" groups respectively. A diagnosis of ACS was made in $10.83 \%$ and $63.3 \%$ of patients in the "observation" and "rule-in" groups respectively.There was a significant reduction in the proportion of patients admitted $27.9 \% \quad \mathrm{v} \quad 20 \%$ with an increase in those admitted being given a primary 
cardiac diagnosis at discharge $52.4 \%$ v 71.7\%. For patients triaged to the "rule out" group and discharged home from the ED there were no MACE (1 MACE in the rule in group appropriately discharged after cardiology review) and no deaths in April 2018 compared with 2 MACE and no deaths in April 2017. For patients in April 2018 with an initial troponin $<5 \mathrm{ng} / \mathrm{L}$ and discharged home there were no MACE or deaths at 30 days.

Conclusion Our results have shown a significant reduction in the median time from ED arrival to discharge, a benefit greatest seen in those presenting with a To value $>12 \mathrm{ng} / \mathrm{L}$. A greater number of patients were discharged from the ED. In those admitted there was an increased PPV for a cardiac diagnosis on discharge. The new pathway was also shown to be safe.

In an NHS era of overwhelmed EDs, 4 hour targets and high inpatient bed occupancy our results have shown the new $0 / 1 \mathrm{~h}$ troponin pathway to be more efficacious than our previous care. Patients were safely triaged to a quicker discharge, with a reduced proportion of admissions. To continue improving the efficacy of the new pathway we have increased the cut off for To to $5 \mathrm{ng} / \mathrm{L}$ in January 2019 and plan to further reassess this pathway.

Conflict of Interest None

\section{VALIDATION OF THE ACS2 SCORE FOR PREDICTING THE PRESENCE OF AN ACUTE CORONARY LESION IN PATIENTS FOLLOWING OUT OF HOSPITAL CARDIAC ARREST}

${ }^{1}$ Matthew Kelham*, ${ }^{1}$ Timothy A. Jones, ${ }^{1}$ Krishnaraj Rathod, ${ }^{1}$ Oliver Guttmann, ${ }^{1}$ Alastair Proudfoot, ${ }^{2}$ Andrew Wragg, ${ }^{1}$ Andreas Baumbach, ${ }^{1}$ Ajay Jain, ${ }^{1}$ Roshan Weerackody, ${ }^{3}$ Anthony Mathur, ${ }^{3}$ Daniel Jones. ${ }^{1}$ Barts Heart Centre; ${ }^{2}$ Barts Health; ${ }^{3}$ Cardiac Research Centre, St Bartholomew's Hospital, London

10.1136/heartjnl-2019-BCS.148

Introduction European guidelines recommend a primary PCI strategy in patients following out-of-hospital cardiac arrest (OHCA) with ST-segment elevation on the ECG. In those without ST elevation the decision to proceed to urgent angiography is recommended if there a high index of suspicion of ischaemia. Predicting which patients are likely to have an acute coronary lesion utilising clinical characteristics available at the time of resuscitation may help inform decision making over which patients should proceed to emergent angiography. The ACS2 score has been shown in a registry of 247 patients to predict the presence of an acute coronary lesion in those resuscitated from an OHCA and hence those who may benefit from emergency coronary angiography. It includes: preceding Angina, Congestive heart failure symptoms, a Shockable rhythm and ST-elevation. We sought to validate this scoring system with an independent cohort of OHCA patients.

Methods We retrospectively analysed patients admitted to our cardiac tertiary centre with an OHCA who underwent coronary angiography between 2014 and 2018 and followed them up until discharge or death in hospital. Baseline demographic characteristics and angiographic findings were recorded.

Results 395 patients were analysed over a 4 year period. The average age was 61.7 years $(\mathrm{SD} \pm 13.9)$. $76.7 \%$ of patients were found to have an acute (culprit) coronary lesion and
$64.3 \%$ of patients had ST-elevation. There was no difference in mean age in those with vs without an acute lesion (62.3 vs 59.1 years, $\mathrm{p}=0.053$ ). Likewise, the presence of an acute lesion was not associated with (percentage with acute lesion): male gender $(76.9 \%$ vs $75.9 \%, \mathrm{p}=0.840)$, existing coronary artery disease $(72.4 \%$ vs $77.7 \%, \mathrm{p}=0.329)$, diabetes $(76.1 \%$ vs $77.8 \%, \mathrm{p}=0.739)$, smoking $(81.4 \%$ vs $77.8 \%, \mathrm{p}=0.486)$, previous coronary intervention $(71.8 \%$ vs $77.7 \%, \mathrm{p}=0.411)$ or previous CABG $(61.1 \%$ vs $78 \%, \mathrm{p}=0.096)$. Similarly, there was no difference in mean downtime (23.6 min vs $22.5 \mathrm{~min}$, $\mathrm{p}=0.071)$ or in-hospital mortality $(35.8 \%$ vs $41.8 \%, \mathrm{p}=0.302)$ in those with vs without an acute lesion.

When the components of the ACS2 model were analysed independently, the presence of an acute lesion was associated with: preceding angina $(87.2 \%$ vs $72.6 \%, p=0.002)$, a shockable rhythm $(80.1 \%$ vs $62.5 \%, \mathrm{p}=0.003)$ and ST-elevation (93.6\% vs $46.4 \%, p=0.0001)$, but not with congestive heart failure symptoms $(79.2 \%$ vs $74.9 \%, p=0.255)$. When analysed with stepwise logistic regression, the model as a whole correctly predicted $82.5 \%$ of cases and had good discrimination (C-statistic 0.832, 95\% CI 0.747-0.858, Hosmer and Lemeshow $\mathrm{p}=0.902)$. The model was superior to the presence of ST-elevation alone (C-statistic 0.802, 95\% CI 0.847-0.858, $\mathrm{X} 2$ difference in -2loglikelihood 18.8, $\mathrm{p}=0.001$ ).

When all factors were given one point, other than ST-elevation which was given two points, and scores were totalled, the percentage of patients found to have an acute coronary lesion was (0-5): 14.3\%, 41.9\%, 54.0\%, 90.6\%, 94.7\% and 95.8\%.

Conclusion These results are the first to our knowledge to validate, using an independent contemporary cohort, the ACS2 scoring system for the prediction of an acute coronary lesion in those resuscitated from an OHCA. As such, our study supports the use of ACS2 to determine which OHCA patients should receive emergency coronary angiography.

Conflict of Interest Nil

\section{CIRCULATING SERUM EXTRACELLULAR MATRIX DEGRADATION ENZYME CATHEPSIN S PREDICTS MORTALITY AND IMPROVES RISK STRATIFICATION OVER THE GRACE SCORE IN PATIENTS WITH NON-ST ELEVATION ACUTE CORONARY SYNDROMES}

${ }^{1}$ Nikolaos Vlachogiannis*, ${ }^{1}$ Kateryna Sopova, ${ }^{2}$ Georgios Georgiopoulos, ${ }^{3}$ Matthias MuellerHennessen, ${ }^{4}$ Marco Sachse, ${ }^{1}$ Constantinos Bakogiannis, ${ }^{3}$ Moritz Biener, ${ }^{3}$ Mehrshad Vafaie, ${ }^{1}$ Aikaterini Gatsiou, ${ }^{1}$ Azfar Zaman, ${ }^{3}$ Hugo Katus, ${ }^{5}$ loakim Spyridopoulos, ${ }^{3}$ Evangelos Giannitsis, ${ }^{2}$ Kimon Stamatelopoulos, ${ }^{1}$ Konstantinos Stellos. ${ }^{1}$ Newcastle University; ${ }^{2}$ University of Athens; ${ }^{3}$ University Hospital Heidelberg; ${ }^{4}$ J.W. Goethe University Frankfurt; ${ }^{5}$ nstitute of Genetic Medicine, Newcastle University

\subsection{6/heartjnl-2019-BCS.149}

Introduction Blood-based biomarkers may be useful in the identification of residual risk for death or acute myocardial infarction (AMI) in patients with a previous acute coronary syndrome. Cathepsin S (CTSS) is a lysosomal cysteine protease with potent elastolytic and collagenolytic activity, which plays an important role in cardiovascular disease through extracellular matrix degradation, vasa vasorum development and atherosclerotic plaque rupture. The aim of the present study was to determine the prognostic and reclassification value of baseline 